\title{
Computing the full two-loop gluon Regge trajectory within Lipatov's high energy effective action
}

\section{G. Chachamis*}

Instituto de Física Corpuscular, Universitat de València - Consejo Superior de Investigaciones Científicas, Parc Cientific, E-46980 Paterna (Valencia), Spain

E-mail: grigorios.chachamis@ific.uv.es

\section{Hentschinski}

Physics Department, Brookhaven National Laboratory, Upton, NY 11973, USA

E-mail: hentsch@bnl.gov

\section{J. D. Madrigal Martínez}

Instituto de Física Teórica UAM/CSIC, Nicolás Cabrera 15, UAM, E-28049 Madrid, Spain

E-mail: josedaniel.madrigal@uam.es

\section{A. Sabio Vera}

Instituto de Física Teórica UAM/CSIC, Nicolás Cabrera 15, UAM, E-28049 Madrid, Spain

E-mail: agustin.sabio@uam.es

We discuss computational details of our recent result, namely, the first derivation of the two-loop gluon Regge trajectory within the framework of Lipatov's high energy effective action. In particular, we elaborate on the direct evaluation of Feynman two-loop diagrams by using the Mellin-Barnes representations technique. Our result is in precise agreement with previous computations in the literature, providing this way a highly non-trivial test of the effective action and the proposed subtraction and renormalization scheme combined with our approach for the treatment of the loop diagrams.

XXI International Workshop on Deep-Inelastic Scattering and Related Subject -DIS2013, 22-26 April 2013

Marseilles,France

${ }^{*}$ Speaker. 


\section{Introduction}

A particularly active field in perturbative QCD which addresses both fundamental formal issues and phenomenological applications is the resummation program of the high center-of-mass energy logarithms. The formalism was initiated almost forty years ago by the Balitsky-Fadin-Kuraev-Lipatov (BFKL) equation [1, 2], which emerged within the framework of high energy factorization. Examples of current applications of the high energy factorization to QCD phenomenology can be found in the analysis of dijets widely separated in rapidity $[3,4,5,6]$, the studies of the transverse momentum dependent parton distribution functions in the low $x$ region $[7,8,9]$ and the the study of observables in heavy ion collisions [10]. Their unifying factor is the factorization of QCD scattering amplitudes in the limit of infinitely high center of mass energies, combined with a resummation of large perturbative logarithmic enhanced corrections.

A very powerful tool for the study of high energy scattering amplitudes is proving to be Lipatov's high energy gauge invariant effective action [11], which attempts to reformulate the high energy limit of QCD as an effective field theory of reggeized gluons. While the determination of the high energy limit of tree-level amplitudes is well understood for a long time within this framework [12], it was only recently that progress in the calculation of loop corrections has been achieved. Starting in Ref. [13] and extended in Refs. [14, 15], a scheme has been developed, combining a subtraction procedure to avoid double counting and the regularization and renormalization of high energy divergences. This scheme then allowed to successfully derive jet vertices for both quark and gluon initiated jets at NLO accuracy from Lipatov's high energy effective action. Within this formalism, we were able to address the more challenging project we discuss here: the determination of the two-loop gluon Regge trajectory $[16,17,18]$. The gluon Regge trajectory provides an essential ingredient in the formulation of high energy factorization and reggeization of QCD amplitudes at NLO and was originally derived in Refs. [19, 20] (see also relevant work in Refs. [21, 22, 23]).

Within Lipatov's effective action approach, to compute the gluon Regge trajectory at NLO, one has to calculate two-loop corrections to the reggeized gluon propagator which, in other words means computing two-loop self-energy Feynman diagrams. This step was actually the main bottleneck of the project, for details, we refer the reader to the original publications $[16,17,18]$. Here, we will restrict ourselves to the discussion of the techniques we used to evaluate the two-loop Feynman diagrams.

\section{The computation of the master integrals}

We focus on the gluonic contributions (no quark loops in the diagrams) to the NLO gluon Regge trajectory which are technically more involved. We remind the reader ${ }^{1}$ that we decompose momenta four-vectors not in terms of light-like vectors $n^{ \pm}$but in terms of deformed light-like vectors, $n^{-} \rightarrow n_{a}=e^{-\rho} n^{+}+n^{-}$and $n^{+} \rightarrow n_{b}=n^{+}+e^{-\rho} n^{-}$, where $\rho$ is an external parameter used to regularize longitudinal divergencies and the amplitude

\footnotetext{
${ }^{1}$ For details, see Refs. [16, 17, 18].
} 
has to be evaluated in the limit $\rho \rightarrow \infty$. As it turns out, only a subset of diagrams can contribute, namely, those that could be potentially enhanced by a factor $\rho^{k}, k \geq 1$.

We have used the Mathematica package FIRE [24] which is an implementation of the Laporta algorithm [25] to reduce the number and complexity of the initial set of integrals into a smaller set of master integrals through integration-by-parts identities [26]. The generic two-loop master integral in our work can be represented ${ }^{2}$ by

$$
\begin{aligned}
& \operatorname{MI}\left[\alpha_{1}, \alpha_{2}, \cdots, \alpha_{9}\right]=\left(\mu^{4}\right)^{-2 \epsilon} \iint \frac{d^{d} k}{(2 \pi)^{d}} \frac{d^{d} l}{(2 \pi)^{d}} \frac{1}{\left(-k^{2}-i 0\right)^{\alpha_{1}}\left[-(k-q)^{2}-i 0\right]^{\alpha_{2}}\left(-l^{2}-i 0\right)^{\alpha_{3}}} \\
& \times \frac{1}{\left[-(l-q)^{2}-i 0\right]^{\alpha_{4}}\left[-(k-l)^{2}-i 0\right]^{\alpha_{5}}} \cdot \frac{1}{\left(-n_{a} \cdot k\right)^{\alpha_{6}}\left(-n_{b} \cdot k\right)^{\alpha_{7}}\left(-n_{a} \cdot l\right)^{\alpha_{8}}\left(-n_{b} \cdot l\right)^{\alpha_{9}}},
\end{aligned}
$$

where $q$ is the momentum of the reggeized gluon whereas $\xi=n_{a}^{2}=n_{b}^{2}=4 e^{-\rho}, \delta=n_{a} \cdot n_{b} \sim 2$.

Dropping all terms that cannot give contributions to the NLO gluon Regge trajectory, we finally have to compute six master integrals, $\mathcal{A}$ to $\mathcal{F}$, which can be seen in Tab. 1, each uniquely defined by the powers of its propagators, along with a certain pre-coefficient associated to each one of them.

\begin{tabular}{c|c} 
master integral & coefficent \\
\hline $\mathcal{A} \equiv[1,1,1,1,0,1,0,0,1]$ & $c_{\mathcal{A}}=-\frac{\boldsymbol{q}^{2}}{2}$ \\
$\mathcal{B} \equiv[1,0,0,1,1,0,0,1,1]$ & $c_{\mathcal{B}}=\frac{66+42 \epsilon}{3+2 \epsilon}$ \\
$\mathcal{C} \equiv[1,1,1,1,1,1,0,0,1]$ & $c_{\mathcal{C}}=-\left(\boldsymbol{q}^{2}\right)^{2}$ \\
$\mathcal{D} \equiv[1,0,0,1,1,1,1,1,1]$ & $c_{\mathcal{D}}=-\boldsymbol{q}^{2}$ \\
$\mathcal{E} \equiv[1,1,0,1,1,1,1,-1,1]$ & $c_{\mathcal{E}}=-2 \xi \boldsymbol{q}^{2}$ \\
$\mathcal{F} \equiv[1,1,1,1,1,1,-2,0,1]$ & $c_{\mathcal{F}}=-\xi \boldsymbol{q}^{2}$
\end{tabular}

Table 1: Master integrals and pre-coefficients. There is also a common overall factor $\left(-2 i \boldsymbol{q}^{2}\right) g^{4} N_{c}^{2}$.

To evaluate the master integrals we have used the Mellin-Barnes representations technique (for a review see e.g. [27]). For that purpose, we re-expressed the diagrams in terms of Schwinger parameters so that we could apply the basic Mellin-Barnes formula:

$$
\begin{aligned}
\frac{1}{\left(X_{1}+\cdots+X_{n}\right)^{\lambda}} & =\frac{1}{\Gamma(\lambda)} \frac{1}{(2 \pi i)^{n-1}} \int \cdots \int_{-i \infty}^{+i \infty} d z_{2} \cdots d z_{n} \prod_{i=2}^{n} X_{i}^{z_{i}} X_{1}^{-\lambda-z_{2}-\cdots-z_{n}} \\
& \times \Gamma\left(\lambda+z_{2}+\cdots+z_{n}\right) \prod_{i=2}^{n} \Gamma\left(-z_{i}\right),
\end{aligned}
$$

\footnotetext{
${ }^{2}$ We use dimensional regularization with $d=4+2 \epsilon$.
} 
where the integration contours are such that poles with a $\Gamma\left(\cdots+z_{i}\right)$ dependence are to the left of the $z_{i}$ contour and poles with a $\Gamma\left(\cdots-z_{i}\right)$ dependencies lie to the right of the $z_{i}$ contour. The Mellin-Barnes representation of the generic master integral reads:

$$
\begin{aligned}
\mathcal{S} & =\iint \frac{d^{d} k}{(2 \pi)^{d}} \frac{d^{d} l}{(2 \pi)^{d}} \frac{1}{\left[-k^{2}-i 0\right]^{\alpha_{1}}\left[-(k-q)^{2}-i 0\right]^{\alpha_{2}}\left[-l^{2}-i 0\right]^{\alpha_{3}}\left[-(l-q)^{2}-i 0\right]^{\alpha_{4}}} \\
& \times \frac{1}{\left[-(k-l)^{2}-i 0\right]^{\alpha_{5}}\left(-n_{a} \cdot k-i 0\right)^{\alpha_{6}}\left(-n_{b} \cdot k-i 0\right)^{\alpha_{7}}\left(n_{a} \cdot l-i 0\right)^{\alpha_{8}}\left(-n_{b} \cdot l-i 0\right)^{\alpha_{9}}} \\
& =\frac{-\left(\boldsymbol{q}^{2}\right)^{d-\alpha_{12345}-\frac{\alpha_{6789}}{2}}}{2(4 \pi)^{d}} \prod_{i=1}^{6} \int \frac{d z_{i}}{2 \pi i} \Gamma\left(-z_{i}\right) \frac{\Gamma\left(z_{1234}+\alpha_{345}+\frac{\alpha_{6789}}{2}-\frac{d}{2}\right) \Gamma\left(-z_{4}+\alpha_{2}\right)}{\prod_{j=1}^{9} \Gamma\left(\alpha_{j}\right) \Gamma\left(-2 z_{1}\right) \Gamma\left(-2 z_{6}\right)} \\
& \frac{\Gamma\left(-z_{12345}-\alpha_{345}+\frac{\alpha_{6789}}{2}+\frac{d}{2}\right) \Gamma\left(-z_{1}+z_{2345}+\alpha_{345}-\frac{\alpha_{6789}}{2}-\frac{d}{2}\right) \Gamma\left(-z_{3}+\alpha_{1}\right)}{\Gamma\left(-2 z_{2}-z_{34}-\alpha_{126789}-2 \alpha_{345}+2 d\right)} \\
& \frac{\Gamma\left(-z_{23}-\alpha_{2345}-\frac{\alpha_{6789}}{2}+d\right) \Gamma\left(-z_{24}-\alpha_{1345}-\frac{\alpha_{6789}}{2}+d\right) \Gamma\left(z_{2345}-z_{6}+\alpha_{3458}-\frac{d}{2}\right)}{\Gamma\left(2 z_{234}+z_{5}+2 \alpha_{345}+\alpha_{789}-d\right)} \\
& \frac{\Gamma\left(2 z_{234}+z_{5}+2 \alpha_{345}+\alpha_{89}-d\right) \Gamma\left(-z_{234}+z_{6}-\alpha_{345}+\frac{d}{2}\right) \Gamma\left(z_{2}+\alpha_{12345}+\frac{\alpha_{6789}}{2}-d\right)}{\Gamma\left(-z_{5}+\alpha_{6}\right)} \\
& \frac{\Gamma\left(-z_{23456}-\alpha_{3458}+\frac{d}{2}\right) \Gamma\left(-2 z_{2}-z_{34}-2 \alpha_{34}-\alpha_{589}+d\right) \Gamma\left(z_{23}+\alpha_{3}\right) \Gamma\left(z_{24}+\alpha_{4}\right)}{\Gamma\left(-\alpha_{34589}+d\right)} e^{-z_{16} \rho}
\end{aligned}
$$

where $z_{i j k \ldots}=z_{i}+z_{j}+z_{k}+\ldots$ and $\alpha_{i j k \ldots}=\alpha_{i}+\alpha_{j}+\alpha_{k}+\ldots$

Once we substitute the actual values of the powers of the denominators for each master integral in Eq. 2.3, we determine the integration contours using the Mathematica package MB.m [28] which also serves as our working platform. We then perform an asymptotic expansion in $e^{-\rho}$ to obtain the leading behavior, using MBasymptotics.m [29]. As a next step we resolve the structure of singularities in $\epsilon$, again using MB .m ( the package MBresolve [30] was also used) and finally we proceed to the evaluation of those terms that are $\rho$ enhanced, making use at this stage also of the routine barnesroutines.m [31].

\section{Conclusions}

We have discussed the approach we used in [18] to compute the two-loop Feynman integrals emerging in our calculation of the NLO gluon Regge trajectory. We have used the Mellin-Barnes representations technique which was proved adequate and which we believe will be further used in future calculations within the framework of Lipatov's high energy effective action.

\section{Acknowledgments}

We thank J. Bartels, V. Fadin and L. Lipatov for their constant support with useful discussions. We acknowledge support by the Research Executive Agency (REA) of the European Union under the Grant Agreement number PITN-GA-2010-264564 (LHCPhenoNet), the Comunidad de Madrid through Proyecto HEPHACOS ESP-1473, and MICINN (FPA2010-17747), the Spanish Government and EU ERDF funds (grants FPA2007-60323, 
FPA2011-23778 and CSD2007-00042 Consolider Project CPAN) and by GV (PROMETEUII/2013/007). G.C. acknowledges support from Marie Curie actions (PIEF-GA-2011298582). M.H. acknowledges support from the U.S. Department of Energy under contract number DE-AC02-98CH10886 and a "BNL Laboratory Directed Research and Development" grant (LDRD 12-034).

\section{References}

[1] L. N. Lipatov, Sov. J. Nucl. Phys. 23 (1976) 338; E. A. Kuraev, L. N. Lipatov, V. S. Fadin, Phys. Lett. B 60 (1975) 50, Sov. Phys. JETP 44 (1976) 443, Sov. Phys. JETP 45 (1977) 199; Ia. Ia. Balitsky, L. N. Lipatov, Sov. J. Nucl. Phys. 28 (1978) 822.

[2] V. S. Fadin, L. N. Lipatov, Phys. Lett. B 429 (1998) 127 [hep-ph/9802290]; M. Ciafaloni, G. Camici, Phys. Lett. B 430 (1998) 349 [hep-ph/9803389].

[3] A. Sabio Vera, Nucl. Phys. B 746 (2006) 1 [hep-ph/0602250]; A. Sabio Vera and F. Schwennsen, Nucl. Phys. B 776 (2007) 170 [hep-ph/0702158]; Phys. Rev. D 77 (2008) 014001 [arXiv:0708.0549 [hep-ph]]; C. Marquet and C. Royon, Phys. Rev. D 79 (2009) 034028 [arXiv:0704.3409 [hep-ph]]; M. Deak, F. Hautmann, H. Jung and K. Kutak, JHEP 0909 (2009) 121 [arXiv:0908.0538 [hep-ph]]; M. Deak, F. Hautmann, H. Jung and K. Kutak, Eur. Phys. J. C 72, 1982 (2012) [arXiv:1112.6354 [hep-ph]]; M. Angioni, G. Chachamis, J. D. Madrigal, A. Sabio Vera; Phys. Rev. Lett. 107 (2011) 191601 [arXiv:1106.6172 [hep-th]]; G. Chachamis, A. Sabio Vera and C. Salas, Phys. Rev. D 87, 016007 (2013) [arXiv:1211.6332 [hep-ph]].

[4] K. Dusling and R. Venugopalan, Phys, Rev. D 87 (2013) 051502 [arXiv:1210.3890 [hep-ph]].

[5] D. Colferai, F. Schwennsen, L. Szymanowski and S. Wallon, JHEP 1012 (2010) 026 [arXiv:1002.1365 [hep-ph]],

[6] F. Caporale, D. Yu. Ivanov, B. Murdaca and A. Papa [arXiv:1211.7225 [hep-ph]], F. Caporale, B. Murdaca, A. Sabio Vera and C. Salas, arXiv:1305.4620 [hep-ph].

[7] H. Jung, S. Baranov, M. Deak, A. Grebenyuk, F. Hautmann, M. Hentschinski, A. Knutsson and M. Kramer et al., Eur. Phys. J. C 70 (2010) 1237 [arXiv:1008.0152 [hep-ph]]; H. Jung and F. Hautmann, arXiv:1206.1796 [hep-ph]; F. Hautmann, M. Hentschinski and H. Jung, Nucl. Phys. B 865 (2012) 54 [arXiv:1205.1759 [hep-ph]]; A. V. Lipatov and N. P. Zotov, Phys. Lett. B 704, 189 (2011) [arXiv:1107.0559 [hep-ph]].

[8] J. Ellis, H. Kowalski and D. A. Ross, Phys. Lett. B 668 (2008) 51 [arXiv:0803.0258 [hep-ph]], H. Kowalski, L. N. Lipatov, D. A. Ross and G. Watt, Eur. Phys. J. C 70 (2010) 983 [arXiv:1005.0355 [hep-ph]].

[9] M. Hentschinski, A. Sabio Vera and C. Salas, Phys. Rev. Lett. 110 (2013) 041601 [arXiv:1209.1353 [hep-ph]], Phys. Rev. D 87 (2013) 076005 [arXiv:1301.5283 [hep-ph]].

[10] B. Schenke, P. Tribedy and R. Venugopalan, Phys. Rev. C 86, 034908 (2012) [arXiv:1206.6805 [hep-ph]]; K. Kutak and S. Sapeta, Phys. Rev. D 86, 094043 (2012) [arXiv:1205.5035 [hep-ph]]; J. L. Albacete, A. Dumitru, H. Fujii and Y. Nara, Nucl. Phys. A 897, 1 (2013) [arXiv:1209.2001 [hep-ph]].

[11] L. N. Lipatov, Nucl. Phys. B452 (1995) 369 [hep-ph/9502308], Phys. Rept. 286 (1997) 131 [hep-ph/9610276]. 
[12] E. N. Antonov, L. N. Lipatov, E. A. Kuraev and I. O. Cherednikov, Nucl. Phys. B 721, 111 (2005) [hep-ph/0411185].

[13] M. Hentschinski and A. Sabio Vera, Phys. Rev. D 85, 056006 (2012) [arXiv:1110.6741 [hep-ph]].

[14] G. Chachamis, M. Hentschinski, J. D. Madrigal and A. Sabio Vera, Phys. Rev. D 87, 076009 [arXiv:1212.4992 [hep-ph]].

[15] M. Hentschinski, Nucl. Phys. B 859, 129 (2012) [arXiv:1112.4509 [hep-ph]].

[16] G. Chachamis, M. Hentschinski, J. D. Madrigal and A. Sabio Vera, Nucl. Phys. B 861, 133 (2012) [arXiv:1202.0649 [hep-ph]]; G. Chachamis, M. Hentschinski, J. D. Madrigal and A. Sabio Vera, arXiv:1206.5101 [hep-ph].

[17] G. Chachamis, M. Hentschinski, J. D. Madrigal and A. Sabio Vera [arXiv:1211.2050 [hep-ph], to appear in Phys. Part. Nucl.].

[18] G. Chachamis, M. Hentschinski, J. D. Madrigal Martínez, A. Sabio Vera, "Gluon Regge trajectory at 2-loop from Lipatov's high energy effective action", article in preparation.

[19] V. S. Fadin, R. Fiore, M. I. Kotsky, Phys. Lett. B387 (1996) 593 [hep-ph/9605357].

[20] V. S. Fadin, R. Fiore and A. Quartarolo, Phys. Rev. D 53, 2729 (1996) [hep-ph/9506432]; M. I. Kotsky and V. S. Fadin, Phys. Atom. Nucl. 59, 1035 (1996) [Yad. Fiz. 59N6, 1080 (1996)]; V. S. Fadin, M. I. Kotsky and R. Fiore, Phys. Lett. B 359, 181 (1995).

[21] J. Blümlein, V. Ravindran and W. L. van Neerven, Phys. Rev. D 58, 091502 (1998) [hep-ph/9806357].

[22] I. A. Korchemskaya and G. P. Korchemsky, Phys. Lett. B 387, 346 (1996) [hep-ph/9607229].

[23] V. Del Duca and E. W. N. Glover, JHEP 0110, 035 (2001) [hep-ph/0109028].

[24] A. V. Smirnov, JHEP 0810 (2008) 107 [arXiv:0807.3243 [hep-ph]].

[25] S. Laporta, Int. J. Mod. Phys. A 15 (2000) 5087 [hep-ph/0102033].

[26] K. G. Chetyrkin and F. V. Tkachov, Nucl. Phys. B 192, 159 (1981).

[27] V. A. Smirnov, Feynman Integral Calculus. Springer, Berlin (2006).

[28] M. Czakon, Comput. Phys. Commun. 175, 559 (2006) [hep-ph/0511200].

[29] M. Czakon, MBasymptotics.m, http://projects.hepforge.org/mbtools/.

[30] A. V. Smirnov and V. A. Smirnov, Eur. Phys. J. C 62, 445 (2009) [arXiv:0901.0386 [hep-ph]].

[31] D. A. Kosower, barnesroutines.m, http://projects.hepforge.org/mbtools/. 